Treatment: cauterization with the nitrate of mercury; the brush was brought several times over the ulcerated surface; slightly astringent injections, baths, rest in bed, very light diet.-The lencorrhceal discharge was streaked with bloc d for a day or two after the cauterization. The latter was repeated every week, and the same treatment, with slight modifications, pursued until she was discharged on the 15th of July. The intensity of the in.. flammatory symptoms soon subsided; she was only confined to her bed for fifteen days, and then allowed to sit up in a recumbent posture. As soon as the ulceration began to cicatrize, the induration diminished, leaving first the posterior plane of the uterus, and the pains and heaviness disappeared, except when walking. As the engorgement diminished, the cervix ascended, ceasing to press on the vagina. On the 15 th of July, the ulceration was quite cicatrized; the cervix had recovered its normal size and colour, the general health was good, and all uneasy sensations had disappeared.

In this instance it is quite evident to me that the origin of the disease may be traced to the last labour; although it was only when it had progressed sufficiently to interfere with menstruation that it attracted the attention of the patient. The slight pains and uneasiness which she, no doubt, had previously experienced, were not perceived, as was the case with Octavie, (Case 3.) A woman who has just gone through all the uneasy sensations of pregnancy and parturition, may well not remark the slight incipient symptoms of ulceration commencing in the cavity of the os uteri. The cases which I next intend to give will be illustrative of more severe forms of the disease.

Cambridge-square, Hyde-park.

\section{PROCESS FOR THE DETECTION OF ARSENIC.}

\section{By H. Letheny, M.B., Lecturer of Chemistry at the Medical School of the London Hospital.}

OF the many processes which have from time to time been suggested, and more or less lauded, too, for the detection of arsenic in cases of poisoning, I am confident that but few of them have been put to the test of experiment with organic mixtures, by way of determining their applicability and delicacy, and the manipulator who is accustomed to seek for arsenic, often in very small quantity, in the contents o: the stomach, or in the tissues of poisoned animals, has learned to look with distrust upon every new suggestion, relying more upon his own tact with regard to some particular process to which he is accustomed, than caring to re-educate himself by adopting any new one. I put forward, however, the following observations, by reason of some little experience in this department of chemistry, and I am convinced, to speak in a general way, that the apparatus, described in TaE LANCET of February 1st, and which was proposed by Berzelius, for the detection of arsenic in cases of poisoning, is not at all applicable, and more particularly in the hands of a novice; for you cannot by that means combat the frothing of the liquor, or prevent small particles of the zine escaping from the central tube into the acid liquor without, and so liberating and losing the arseniuretted gas. Again : to be certain of decomposing this gas, the exit pipe must be heated from the commencement, and if care has not been taken to expel the air, it will assuredly explode. Moreover, there is tact required in the application of the heat, or we either get the tube softened and bend. ing, and perhaps blowing out, or the arsenium, by the great heat, is carried over, or else the gas is not decomposed at all; and if it so happen that the tuhe is a little too small, or gets closed up by a film of arsenium, the gas then bubbles through the acid liquor and is lost, and we cannot make a quantitative analysis: all these are difficulties in the way even of the experienced, but they are most formidable to the novice.

In place of this process, which I have long given up, I adopt the following, which is a modification of Lassaigne and Reinsch, and I never find the least difficulty in detecting even very small quantities of arsenic. Supposing $I$ have the contents of a stomach to test. They are first boiled in distilled water, slightly acidulated with acetic acid, then filtered, and again and again boiled and filtered; mix these liquors and divide into two equal parts. Evaporate one part nearly to dryness, and then heat with about twice its bulk of sulphuric acid, until it is quite charred, and begins to evolve the acid. This mixture is then to be diluted and introduced, by little and little, into a large Wolfe's bottle, from which hydrogen is being slowly evolved from pure zinc and dilute sulphuric acid. The gas is to be conveyed by means of a bent tube, drawn fine at its remote point, to the bottom of a tall glass, containing a solution of nitrate of silver; it will occasion a black precipitate in it, and by taking the precaution of not allowing the gas to come over too quickly, and by means of the fine tube to make it pass through in very small bubbles, we shall be certain to decompose the whole of it. To this black turbid solution of nitrate of silver is then to be added muriatic acid, until all the silver is precipitated, and a little of the acid remains in excess; boil for a few minutes, and filter and evaporate the filtered liquor to dryness; the residue, if there be any, is to be dissolved in a little distilled water, and carefully precipitated by ammoniacal nitrate of silver; if any arsenic had been present, it would by this process have been converted into arsenic acid, and this with the ammoniacal nitrate would produce the red precipitate of arseniate of silver, every $464 \mathrm{grs}$. of which is equivalent to $100 \mathrm{grs}$. of arsenious acid, or 76 of arsenicum. This arseniate of silver may be again reduced by means of black flux, or charcoal, and preserved for evidence. The other half of the suspected liquor is to be slightly acidulated with muriatic acid, and boiled for an hour or two with a known weight of clean copper in strips; if arsenic is present, it will attach itself to the metal, g!ving it a black coating. The copper being removed, dried, and weighed, indicates how much heavier it may have hecome, and to be certain of this being arsenic, they should be placed in a small green glass tube, closed at one end, and by means of a spirit lamp, with a large flame or a blow-pipe, kept at a red heat for a little time; if arsenic be there, it will sublime either as a white or black ring. After this, the copper is to be again weighed and its loss estimated.

Supposing, however, that the substance to be tested were a piece of muscle or liver. It is to be charred by means of sulphuric acid, as in the first case, and the liquor diluted and divided into two portions; the one to be treated by Lassaigne's process with the nitrate of silver; the other to be nearly neutralized by carbonate of soda, and boiled, as in the last case, with the clean copper.

Now the advantages of these processes are, that they are readily applicable and will detect small quantities. The frothing is obviated, and you are not likely to be annoyed by the fallacies which antimony may occasion. Not but that antimony will decompose the nitrate of silver, and be deposited on the copper like arsenic; but then the subsequent stage of the processes determines this.

In conclusion, I may mention as a caution, that there is again in the market a large quantity of sulphuric acid contaminated with arsenic; I have found, in a recent sample, as much as one grain in the fluid ounce.

Tredegar-square, Jan. 6,1845 .

\section{ON THE PECUIIAR CREPITUS OF THE LUNGS} AFTER PLEURISY.

By Rrchard Crambers, Esq. Colchester. To the Editor of THE LA NCET.

SrR,--In consequence of your observations in THE LANCET of the 8th instant, I am induced to send you the annexed case, believing that it possesses some value in deciding the seat of this sign. I have the honour to be, your obedient servant,

Colchester, March 10, 1845.

Richapd Chambers.

WILLIAM I-, aged nineteen, was admitted, April 13th, 1843, into the Essex and Colchester Hospital, labouring under symptoms of chronic pleuritis of the left side; the right lung appeared everywhere healthy, except in the lateral region, where a fine crepitus could be heard over a space of about two inches in diameter; I believed it to have been eaused by a circumscribed pneumonia, and was surprised at the absence of all other symptoms of that disease. I mentioned the case at a meeting of our medical society, calling attention to its unusual character. Leeches and blisters were applied over the part affected, but they produced no alteration in the crepitus, which continued distinct during the period of three months that he was under observation.

He became subject to attacks of cerebral congestion, and as his constitution was evidently sinking, he left the hospital on the 3rd of July, and died at his own house on the 22nd of the same month. We obtained a post-mortem examination the next day, which was carefully performed by Mr. Partridge, jun.

The left lung was closely adherent to the ribs, and in that portion of the right side where the crepitus existed, we found shreds of lymph connecting both pleuræ. The lung itșelf was perfectly healthy.

I entertained no doubt that the crepitus arose from the friction of the lung against the pleura, the surface being rendered rough by the intervention of the bands of lymph; although, during repeated examinations, I never once doubted the similarity between the crepitus and that caused by pneumonia. The case was also examined by Dr. Bowtree, now staff assistant-surgeon, and $\mathrm{Mr}$. Baker, then house-surgeon, both of whom agreed in the opinion I had formed. 
As crepitus is merely a physical sign, and can be excited under very dissimilar circumstances, we can easily understand, and ought as readily to admit, that it may be imitated by the friction of bands of lymph between the lung and pleura; and this view of the subject acquires confirmation from the method resorted to by Professor Williams to convey a correct notion of the sound-viz., rubbing a lock of hair between his fingers.

The advocates of other explanations of this sign may urge that a friction sound has been mistaken for that of crepitation; this I readily admit, and even more than that, having since then had frequent opportunities of hearing the sound in cases where I knew that it arose from a pleural friction. I confess myself unable to distinguish any difference between the crepitus of pneumonia and that which arises in some cases of pleuritis, when viewing them apart from the other symptoms of those diseases; and in this opinion $I$ think $I$ am corroborated by an accomplished auscultator, Dr. F. Ward, of Kensington, at least, I beg particularly to refer my readers to a case published by him in the Medical Gazette, of Nov. 1, 1844, in which he states that a moist crepitation could be heard over a portion of lung, free from other traces of disease, and in which, after death, the lung and pleura were found to be connected by bands of lymph. I consider the case particularly interesting, as it affords unbiassed and convincing testimony to the correctness of my views. It is true that he gives a different explanation of the cause, but should this meet his eye, I think he will be disposed to alter his opinion, which is, that the crepitus arose from codema of the lung, but this, as he remarks, was only local, and, more probably than not, was a post-mortem appearance.

The explanation I have adrocated explains the sign on known grounds, and does not require the adoption of theory.

\section{NOTES IN PRIVATE PRACTICE.}

By S. Grower, Esq., Surgeon, late Medical Officer to the Holme and Woolden Districts, Huddersfield Poor-law Union.

HERNIA.

When persons are thronging out of a theatre, one pressing another on, and each struggling to get first through a narrow opening which will permit but one at a time to pass, the theatre does not get emptied so soon as if all agreed to go out at such a pace as to leave the passage free for an uninterrupted succession of one after another going through it quietly. If two stout persons, forced on by the crowd behind, should get wedged into that narrow passage, so that neither could stir, the obstruction, so long as it should remain unremoved, would be total, and prevent all egress to those behind. In the reduction of hernia by the taxis something not very dissimilar happens, from too violent pressure being made on the hernial tumour. I have seen violent efforts made at reduction, causing great pain to the patient; such efforts remitted, then repeated, to the injury of the contents of the sac, and then an operation talked of as the only method left. I have made gentle but firm pressure in the proper direction, just enough not to cause much pain. I have maintained such pressure, not relaxing it for a moment, but when one hand became wearied, substituting the other, till I have had the satisfaction of feeling the bowels slipping quietly, and almost imperceptibly, into their place, till the sac has been emptied. It may be set down as one rule of guidance, that you are not to make such pressure as will cause severe pain, for you thus not only cause pain, and do mischief to the parts, but crowd up and obstruct the narrow pass through which the bowels have to return. On making the requisite degree of pressure only, and patiently continuing it, a little air, or other contents of the intestine, will slip up, and a little bit of bowel with it, till you find the whole contents of the sac giving way under your hand. When this is perceived, the degree of pressure should be rather increased than diminished; but at any rate no relaxation of pressure must take place after the whole body of the hernia is felt to be on the move, till every fraction is returned. No good can be done by kneading a hernial tumour about like dough.

\section{PHYMOSIS.}

I have met with a great many cases of phymosis on which I have had to operate. I have seen part of the glans sloughing away at a dreadful rate, for want of laying the prepuce open sufficiently early. But I wish to direct attention to this case chiefly as it is met with in children. The passage of the urine is sometimes obstructed by a congenital redundancy of prepuce. Infants may be observed to cry before and during the emptying of the hladder, and the prepuce will be found longer than usual, and with an orifice too small to admit, or to admit easily, of its being drawn back upon the glans, so as to expose the meatus. There is sometimes an adhesion of the inner part to the glans, not of an organized kind, but they are glued together by the natural secretions. In these cases the child suffers much inconvenience and pain; but actual circumcision, though generally a harmless operation, is not often necessary. If the nurse draws back the prepuce gently over the glans twice or three times a day, and uses warm water ablutions freely, the case will soon cease to require attention. Other cases require an operation on the prepuce, and circumcision is preferable to any other. This may seem a slight and easy operation, but owing to the slippery nature of the parts, and the double-nightcap shape of the prepuce, it is possible to perform it very awkwardly and ineffciently. You may cut off what seems a sufficient piece of prepuce, and find that you have only denuded the part by the abstraction of a circumference of the outer fold, leaving an ugly. looking sore, and the meatus not freed, or insufficiently freed, from obstruction.

I have operated in various ways, but the following is the mode to which I shall hereafter adhere:-Having pulled forward the prepuce, everting as much as possible the inner fold, and placed it, as on a block, on the smooth surface of a soft piece of cork, of sufficient size, put a thimble on, and force a stout common needle through all the four layers of membrane at the end of the prepuce into the cork; when thus fixed, draw out the prepuce with a moderate tenseness away from the glans, by means of the needle and piece of cork, and cutting down upon the cork, remove the requisite portion of prepuce. Neither the tenaculum nor the forceps, nor the fingers, are preferable to the common needle thus used, whether as regards the pain caused by, or the time taken in, the operation, or the precision of the result

In the last case of this kind that occurred to me, the bladder had been supposed to be the seat of the disease, and I had the satisfaction both of detecting and curing an ailment which had caused some years of uneasiness and suffering. Sometimes the prepuce is not redundant, but fitting tensely upon the glans, and having only a pin's-head orifice, which will scarcely admit of a probe, and that orifice not situated correspondingly to the meatus of the glans, but on one side of it. In these cases a wide slit is perhaps preferable. When a mother or nurse complains of a male child's crying before micturition, the prepuce should always be examined.

ON THE MODE OF INDUCING PREMATURE LABOUR.

We are informed, in works on midwifery, that in rupturing the membranes for the purpose of bringing on premature labour, a catheter is preferable to a sound because the liquor amnii flowing through the former gives more distinct notice of the rupture having been effected. I have found this expectation fallacious, and believe it would frequently be found so. The os ateri is commonly spoken of as being sealed up by a mucous secretion. That such a secretion gravitates towards the os uteri, and collects there, is certain, but that it acts in any manner as a seal seems more than disputable; a person would be sorry to send a letter to the post, especially in these latter days, that was not better wafered or sealed. This mucous secretion is, however, viscid enough to stop up the hole or holes of the catheter, so as to deprive it of the power of intimating any more than a sound would the successful rupture of the membranes, besides misleading the operator, who expects such intimation from it A catheter, with a plug at the end of the stilette, shaped like the heater of an Italian iron, which should wholly exclude the intromission of this secretion during its passage through the os and which could be withdrawn on the supposed penetration of the membranes, would answer better; and there are other cases in which catheters, thus provided with a plug, would be useful. But in using either catheter or sound some inconvenience is experienced, owing to the distortion which renders the operation necessary, and a leaden sound, flexible enough to be adapted to the curve required, by hending it, and firm enough to penetrate the membranes, (this, of course, and not to break or bend too much, would be preferable to either. Such an instrument ought to be rather more than the full length of a male catheter. There is more occasion to be careful in introducing the instrument into the uterus than might be supposed by a person who had not tried it.

A mode of producing premature labour has been recommended, which renders the rupture of the membranes by an instrument, and indeed their rupture at all, unnecessary. The os uteri is to be teased and irritated for half an hour at a time, twice a day, for some days together, by the introduction and twisting about of the finger. Some thirty years ago there was a contention as to the priority of claim to the discovery of this method hetween my old master, the late Mr. Jones, of Finsburysquare, and another practitioner. Mr. Jones certainly was the first in order of time to discover, and I believe also to recommend it; and he practised it himself with success in a very bad case. He saved both child and mother by it. I nevertheless 